\title{
The International Association of Oral \& Maxillofacial Surgery: An Organization of Contrasts and Similarities
}

\author{
Larry Nissen
}

Published online: 10 March 2011

(C) Association of Oral and Maxillofacial Surgeons of India 2011

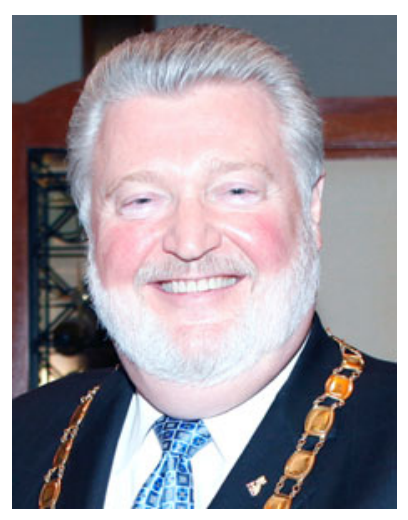

The International Association of Oral and Maxillofacial Surgeons is an organization of 75 affiliated nations and approximately 5000 members. It is charged with elevating the quality of healthcare worldwide through the advancement of the art and science of oral and maxillofacial surgery. The IAOMS works to achieve its goals through providing an educational, professional and social forum for oral and maxillofacial surgeons of the world, allowing all within our specialty to become an integral part of a unique organization.

The educational programs of the IAOMS range from basic week-long courses in various locations over an 18 to 24 month period, to year-long Fellowship training programs in subspecialty areas of Cleft/Craniofacial Surgery and Oncology/Reconstructive Surgery, to intense training in Microsurgical techniques. The recently added electronic learning opportunities augments the Association's educational programs which is highlighted by the bi-annual

L. Nissen $(\square)$

280 N. Sykes Creek Parkway \#C, Merritt Island, FL 32953, USA

e-mail: jwsrgn@aol.com

International Conference that brings together the best and the brightest of the maxillofacial "world" to present the latest in science in our ever expanding realm of healthcare.

New endeavors for the Association include investigating the feasibility of establishing an IAOMS training program accreditation process and an IAOMS "Board" examination, both focused on elevating the educational levels of OMS in all parts of the world, particularly those areas that do not currently have these opportunities. There is also a group developing a Disaster Relief Team, to respond to areas in the world that experience mass disasters that result in multiple maxillofacial injuries, such as the earthquakes in Haiti and Chile. An Informational Management Committee has been established that will advise the Board and Executive Committee on matters of technology and communication, the importance of which cannot be over emphasized in years ahead.

The future of the IAOMS: collaboration and communication; both with members of our specialty and nonmembers, and not just healthcare providers, but with allied groups and industry. Expansion of our specialty's knowledge base is critical for progress and we cannot ignore the importance of research, both laboratory and clinical. To remain relevant, the specialty MUST move forward. To move forward, we must have leaders that look to prospectively with a view and respect of the past. The IAOMS is THE organization help mold the future of our specialty in a global sense, understanding and respecting individual, national and regional differences; but working for a common cause: making oral and maxillofacial surgery THE premier healthcare specialty. 